\title{
Location-based Media Sharing in a MP2P Network
}

\section{Niko Kotilainen $^{b} \quad$ Lito Kriara $^{a} \quad$ Konstantinos Vandikas $^{a} \quad$ Konstantinos Mastorakis $^{a}$ Maria Papadopouli ${ }^{a}$}

\author{
${ }^{a}$ Department of Computer Science, University of Crete, Greece \& \\ Institute of Computer Science (ICS), Foundation for Research and Technology - Hellas (FORTH) \\ ${ }^{b}$ Department of Mathematical Information Technology, University of Jyväskylä, Finland
}

In both academia and industry, peer-to-peer $(p 2 p)$ applications have attracted great attention. This paper introduces and implemented a novel location-based multimedia application, the Multimedia Traveling Journal application (PhotoJournal) that employs the $p 2 p$ paradigm and enables location-based content sharing among mobile users.

\section{Introduction}

The Web and Internet have been catalysts for the creation of collaborative applications and tools. On-line collaboration has been enriched with new applications and tools for sharing and experimenting with multimedia data in a synchronous or asynchronous manner, such as YouTube and Flickr. These technologies have allowed the formation of new types of social networks, interactions, and online communities. We anticipate that in the near future mobile devices that have the processing, communication and geolocating capabilities will enable seamless integration of services combining media sharing and geographical tagging.

The Multimedia Traveling Journal application (PhotoJournal) applies the peer-to-peer (p2p) paradigm to share location-based content among mobile devices. It also enables users to build interactive multimedia journals that associate multimedia objects such as pictures, video, or hypertext, with locations on maps. The PhotoJournal is supported by a middleware with two main components, namely a positioning and an information discovery system. The underlying positioning technologies are GPS and Cooperative Location-sensing System (CLS) [1, 2]. 7DS [3] enables information discovery and sharing in a $\mathrm{p} 2 \mathrm{p}$ manner. Section II focuses on PhotoJournal and briefly introduces CLS and 7DS, while Section III summarizes our main conclusions and future work plans.

\footnotetext{
${ }^{*}$ This work was supported by the Greek General Secretariat for Research and Technology under Programs Regional of Crete,

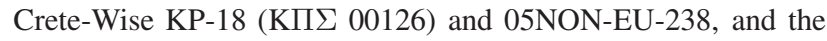
European Commission (MIRG-CT-2005-029186). Niko Kotilainen participated in this project while visiting the University of Crete and FORTH. Contact person: Maria Papadopouli (mgp@ics.forth.gr).
}

\section{Architecture of PhotoJournal}

In general when an application requests a data object, 7DS first checks its cache, and if the data is not available, it tries to acquire it from the Internet. If the local web client fails to connect to the Internet, the local 7DS instance multicasts a query about that object in the wireless LAN. Figure 1 summarizes the main components of the location-based media sharing system, namely the PhotoJournal application, 7DS and CLS.

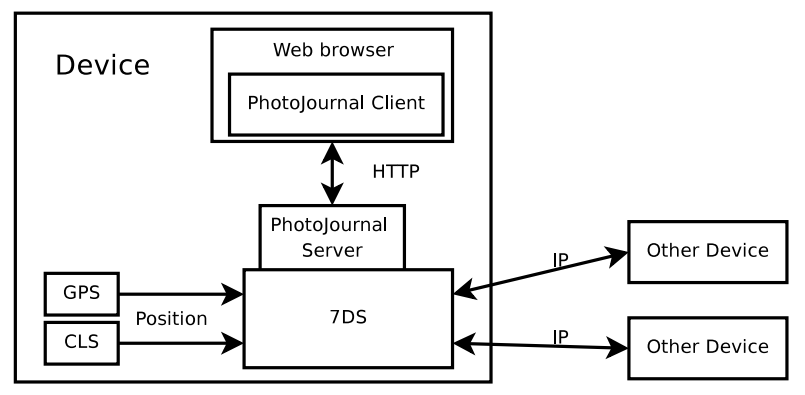

Figure 1: The architecture of a location-based media sharing system.

Through 7DS, PhotoJournal allows peers to share files associated with certain locations. The multimedia files and maps are stored in the cache of the local 7DS instance. A user can add multimedia objects to a certain point of the map by clicking on the map and browsing the image files corresponding to that location. Moreover, the user can add, modify, or delete comments on a certain multimedia file, and rate its content. A multimedia file can be set public or private, while only public files are shared with other peers.

The PhotoJournal searches other 7DS peers for multimedia files associated with a given area marked on the map by the user. It forms a 7DS query and mul- 


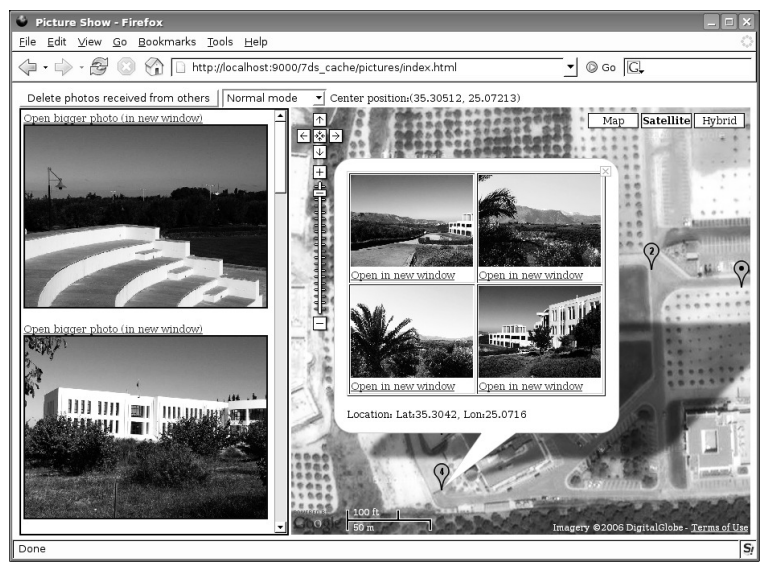

Figure 2: PhotoJournal can superimpose multimedia objects at their locations on a map. A marker indicates the number of files associated with that location.

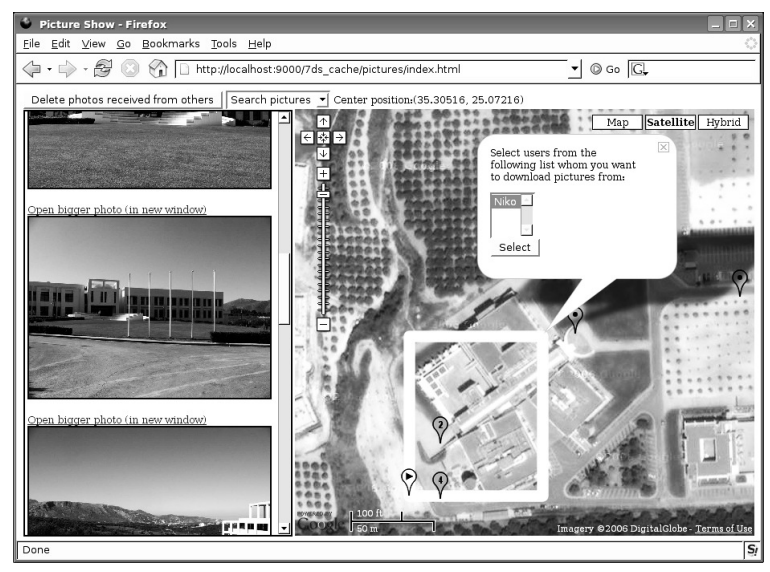

Figure 3: A user can mark the area for which multimedia objects are requested.

ticasts it to other 7DS peers. Furthermore, it maintains and displays a list of neighboring 7DS peers, updating it periodically. A user may then select the 7DS peers from which the application retrieves the files associated with the predefined area, stores them in the local cache and displays them on the map (as illustrated in Figures 2 and 3). Areas on the map associated with multimedia files can be distinguished by a marker that also indicates the number of the available relevant files. Moreover, the queries are formed using location-based or rate-related criteria. The response of a peer includes the multimedia files and reviews.

As shown on Figure 3, a web browser is the frontend of PhotoJournal. It consists of a map frame on the right and a photo bar on the left side of the window. Its backend runs on 7DS. It receives all queries from the frontend through 7DS's proxy server, and supports the typical 7DS functionality by adding or deleting photos, querying photos from 7DS neighbors or handing out photos from the local cache. 7DS can also cache map files, enabling the application to work without an Internet connection.

If the device has a built-in camera, users can take photos and videoclips and upload this information along with position traces (produced by GPS or CLS) to the PhotoJournal which can automatically associate the multimedia files with the user's current position. The PhotoJournal can automatically superimpose the uploaded content on an appropriate map by matching the timestamp of the content of the multimedia files with the timestamp of the GPS/CLS trace. Furthermore it locates them on the map, and updates its local 7DS cache.

CLS is a novel location sensing system that employs the $\mathrm{p} 2 \mathrm{p}$ paradigm and a probabilistic framework to estimate the position of wireless-enabled devices in an iterative manner. CLS can incorporate signal-strength maps of the environment to improve the position estimates. Such maps have been built using measurements that were acquired from access points and peers during a training phase. Periodically, CLS can refine its positioning estimations by incorporating newly received information from other devices.

At run-time, the local CLS instance acquires signalstrength measurements from peers, constructs a runtime signature, and compares it with the ones that have been generated during the training phase. For the comparison, it employs confidence interval-based and percentiles-based criteria.

CLS adopts a grid-based representation of the physical space; each cell of the grid corresponds to a physical position of the physical space. The cell size reflects the spatial granularity/scale. Each cell of the grid is associated with a value that indicates the likelihood that the node is in that cell. These values are computed iteratively using one of the following two approaches, namely a voting algorithm and a particle filter based model.

In the voting process, a local CLS instance casts votes on cells of the grid based on measurements received from peers. A signature associates each cell of the grid with a vector of statistical information of the RSSI values that were recorded from messages received from those peers during the training and runtime phases. The algorithm assigns a weight at each cell depending on the similarity of the training and runtime signatures. The cell with the highest weight is the one that CLS reports as the user's position.

The CLS particle-filter based approach can be formulated in probabilistic terms as the problem of determining the probability of a node being at a certain location given a sequence of signal strengths. Assum- 
ing first-order Markov dynamics, the above problem can be expressed using the network graph depicted in Figure 4 , where $x_{k}$ is the node location (system state) at time instant $k=1, \ldots, T . x_{k}$ cannot be observed directly (it is "hidden"). Yet, for each location $x_{k}$, a measurement vector $y_{k}$ (signal strength) is available that depends on the hidden variable according to a known observation function.

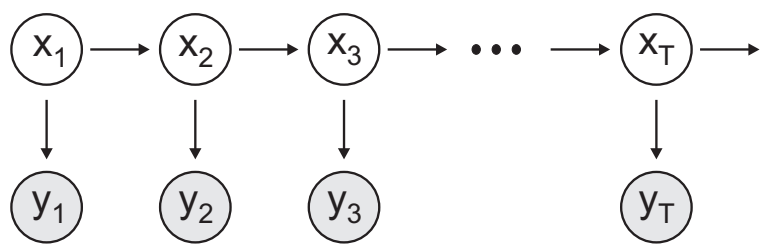

Figure 4: Clear circles indicate hidden state variables, grayed circles indicate observations, horizontal arrows indicate state transition functions and vertical arrows indicate observation functions.

Due to the Markov assumption, each node location, given its immediately previous location, is conditionally independent of all earlier locations, that is $P\left(x_{k} \mid x_{0}, x_{1}, \ldots, x_{k-1}\right)=P\left(x_{k} \mid x_{k-1}\right)$. Similarly, the observation at the $k$-th time instant, given the current state, is conditionally independent of all other states $P\left(y_{k} \mid x_{0}, x_{1}, \ldots, x_{k}\right)=P\left(y_{k} \mid x_{k}\right)$.

Based on the this model, location-sensing can be formulated as the problem of computing the location $x_{k}$ of a node at time $k$, given the sequence of observations $y_{1}, y_{2}, \ldots y_{k}$, up to time $k$, that is, determining the $a$ posteriori distribution $P\left(x_{k} \mid y_{1}, y_{2}, \ldots, y_{k}\right)$, using particle filters. To generate and maintain the particles, we utilize the Sampling/Importance Resampling (SIR) algorithm introduced by Rubin [4]. According to SIR, instead of sampling the true a posteriori distribution (which is not possible because this distribution is not available in closed form), particles are drawn from the so-called proposal distribution $\pi\left(x_{k} \mid y_{1}, y_{2}, \ldots, y_{k}\right)$. To compensate for this difference, each particle $s^{(L)}$ is also assigned a weight $w^{(L)}$, which is computed, according to the Importance Sampling Principle: $w_{t}^{(L)}=\frac{P\left(x_{k} \mid y_{1}, y_{2}, \ldots, y_{k}\right)}{\pi\left(x_{k} \mid y_{1}, y_{2}, \ldots, y_{k}\right)}$

The performance of CLS was evaluated in Telecommunication and Networks Laboratory at FORTH, an area of $7 x 12 \mathrm{~m}^{2}$ and a median location error of $1.8 \mathrm{~m}$ was reported [2].

\section{Conclusions}

This implementation of PhotoJournal has adopted a $\mathrm{p} 2 \mathrm{p}$ architecture. We are implementing a more centralized approach for thin devices (e.g. smart-phones) in which a client acquires and sends the multimedia files to a webserver. The hybrid architecture facilitates both the centralized and p2p approaches, enabling devices to acquire the data either from a web-server or another peer. We are in the process of deploying a testbed in an aquarium and perform user studies. Furthermore, we will evaluate the delay and scalability of these architectures via empirical-based measurements and simulations.

Privacy plays a critical role in the adoption of mobile peer-to-peer computing applications. 7DS-like systems facilitate sharing among devices in different types of environments. However, depending on the application and usage peers may have different privacy requirements. Currently, 7DS offers a crude distinction between private and non-private objects and a finer description of the privacy requirements is required.

Mobile p2p computing is a relatively new technology, not yet proven in the research community and industry. A fruitful approach would be to develop a general infrastructure for mobile peer-to-peer applications, build some robust applications, and extract a toolkit that other new applications could use. Our group sets the directions for exploring this technology further.

\section{References}

[1] C. Fretzagias and M. Papadopouli, "Cooperative location-sensing for wireless networks", Second IEEE International conference on Pervasive Computing and Communications 2004, Orlando, Florida, March 14-17, 2004.

[2] K. Vandikas, L. Kriara, T. Papakonstantinou, A. Katranidou, H. Baltzakis and M. Papadopouli, "Empirical-based analysis of a cooperative location-sensing system", First International Conference on Autonomic Computing and Communication Systems (ACM Autonomics'07), Rome, Italy, October 28-30, 2007.

[3] M. Papadopouli and H. Schulzrinne, "Effects of power conservation, wireless coverage and cooperation on data dissemination among mobile devices", ACM SIGMOBILE Symposium on Mobile Ad Hoc Networking \& Computing (MobiHoc) 2001, October 4-5, 2001, Long Beach, California.

[4] D.B. Rubin, "Using the SIR Algorithm to Simulate Posterior Distributions.", Bayesian Statistics. Oxford University Press. vol.3, p.395-402, 1988. 